\title{
ITERATIVE DENOISING ALGORITHMS FOR PERFUSION C-ARM CT WITH A RAPID SCANNING PROTOCOL
}

\author{
Michael T. Manhart $\quad$ Andreas Fieselmann ${ }^{\star} \quad$ Yu Deuerling-Zheng ${ }^{\dagger} \quad$ Markus Kowarschik $^{\dagger}$ \\ * Pattern Recognition Lab, Friedrich-Alexander-Universität Erlangen-Nürnberg, Germany \\ ${ }^{\dagger}$ Siemens AG, Angiography \& Interventional X-Ray Systems, Forchheim, Germany
}

\begin{abstract}
Tissue perfusion measurement using $\mathrm{C}$-arm angiography systems capable of CT-like imaging (C-arm CT) is a novel technique with potentially high benefit for catheter-guided treatment of stroke in the interventional suite. New rapid scanning protocols with increased $\mathrm{C}$-arm rotation speed enable fast acquisitions of $\mathrm{C}$-arm CT volumes and allow for sampling the contrast flow with improved temporal resolution. However, the peak contrast attenuation values of brain tissue lie typically in a range of 5-30 HU. Thus perfusion imaging is very sensitive to noise. In this work we compare different denoising algorithms based on the algebraic reconstruction technique (ART) and introduce a novel denoising technique, which requires only iterative filtering in volume space and is computationally much more attractive. Our evaluation using a realistic digital brain phantom shows that all methods improve the perfusion maps perceptibly compared to Feldkamptype (FDK) reconstruction. The volume-based technique performs similarly to the ART-based methods: the Pearson correlation of reference and reconstructed blood flow maps increases from 0.61 for the FDK method to 0.81 for the best ART method and to 0.79 for the volume-based method. Furthermore results from a canine stroke model study are shown.
\end{abstract}

Index Terms - Perfusion imaging, iterative reconstruction, C-arm CT, stroke treatment

\section{INTRODUCTION}

Perfusion CT (PCT) is an important imaging modality for diagnosis in case of an ischemic stroke event. Time attenuation curves (TACs) of contrast flow in tissue and vessels are extracted from a time series of brain volumes acquired after a contrast bolus injection. Perfusion parameter maps calculated from TACs, which represent quantities such as cerebral blood flow (CBF), cerebral blood volume (CBV), mean transit time (MTT), and time-to-peak (TTP), provide information about the extent of the affected tissue. They can be used to identify potentially salvageable ischemic tissue that could be reperfused by catheter-guided stroke therapy procedures such as intra-arterial thrombolysis and mechanical clot retrievement. For this purpose the patient is transported to an interventional suite with a $\mathrm{C}$-arm angiography system, where perfusion measurement is commonly not yet available. Perfusion measurement using $\mathrm{C}$-arm systems would allow for assessing the perfusion parameters directly before and during the interventional procedure and help to determine the treatment success and endpoint. Additionally, perfusion C-arm CT (PCCT) can - in contrast to PCT - acquire 3D perfusion maps in high resolution in $\mathrm{z}$ (axial) direction with full brain coverage. However, PCCT is challenging: common C-arm systems typically need $5 \mathrm{~s}$ to acquire one volume, which limits the temporal resolution of the reconstructed TACs. Furthermore perfusion imaging is highly sensitive to noise since the peaks of the TACs inside the brain tissue typically lie in a range of 5-30 HU. Recently, we proposed a dynamic iterative reconstruction technique [1] to reconstruct TACs with increased temporal resolution from the acquired X-ray projections which uses a regularization based on joint bilateral filtering (JBF) [2] to increase the contrast-to-noise (CNR) level of the brain tissue TACs. However, this approach has a much higher computational effort than FDK reconstruction and requires subtraction of the static anatomic structures in projection space. A further possibility to improve the temporal sampling of the TACs is a rapid scanning protocol with increased rotation speed of up to $100 \%$ s using novel robotic C-arm systems (Artis zeego, Siemens AG, Germany). For the rapid scanning protocol we recently applied an iterative tight frame (TF) wavelet-based reconstruction algorithm [3] in a brain perfusion phantom study [4] to increase CNR in the tissue TACs, which showed promising results to improve the perfusion maps compared to FDK [5] reconstruction. In this work, we compare the latter approach to the JBF and the improved total variation (iTV) [6] regularization techniques. However, all these approaches are based on ART [7] and thus computationally expensive. As discussed in [8] analytical reconstruction followed by iterative denoising in volume space can achieve similar results in noise reduction as the ARTbased approaches with much less computational effort. Thus we also developed a pure volume-based denoising technique and show that it provides similar results regarding perfusion measures as the fully iterative algorithms.

The rapid scanning protocol consists of two acquisition sequences: the first sequence acquires one sweep in forward 
and one in backward C-arm rotation before bolus injection to reconstruct baseline volumes with static anatomical structures. The second sequence then acquires $N_{\text {rot }}=7$ consecutive sweeps with alternating forward and backward C-arm rotations after bolus injection to reconstruct a time series of contrast-enhanced volumes. Each sweep acquires 133 projections in a $200^{\circ}$ angular range and requires $T_{\mathrm{r}}=2.8 \mathrm{~s}$ for data acquisition with a pause of $T_{\mathrm{w}}=1.2 \mathrm{~s}$ between the sweeps. Thus TACs can be sampled with a temporal resolution of $T_{\mathrm{s}}=T_{\mathrm{r}}+T_{\mathrm{w}}=4 \mathrm{~s}$.

\section{DENOISING TECHNIQUES}

\subsection{Iterative Algebraic Reconstruction Algorithms}

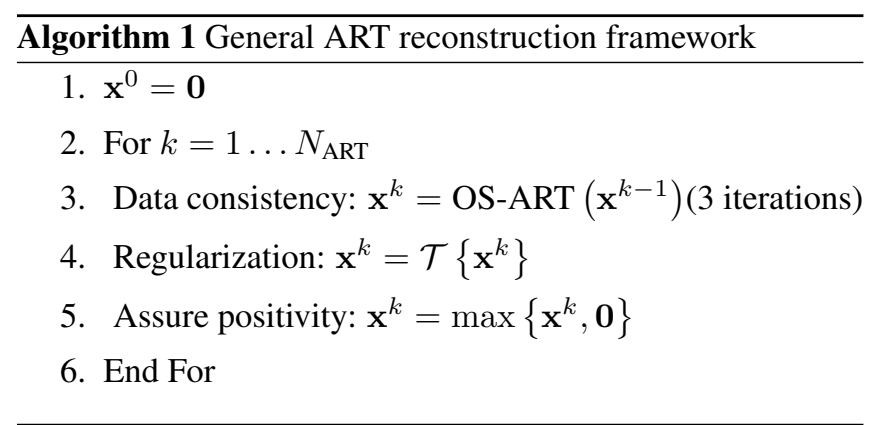

The ART-based methods reconstruct the 3D volume $X \in$ $\mathbf{R}^{3}$ represented as a column vector $\mathbf{x}$ from the measured projection data $\mathbf{p}$ by minimizing:

$$
\underset{\mathbf{x}}{\operatorname{argmin}}\|A \mathbf{x}-\mathbf{p}\|_{2}+\lambda \mathcal{R}(\mathbf{x}) .
$$

The system matrix $A$ describes the cone-beam acquisition geometry of the $\mathrm{C}$-arm system and $\mathcal{R}(\mathbf{x})$ is a penalty term incorporating prior knowledge about volume smoothness, where scalar $\lambda$ controls its influence. Each baseline and contrast-enhanced scan is reconstructed separately. Equation 1 is solved using Algorithm 1 by alternately minimizing the data consistency term $\|A \mathbf{x}-\mathbf{p}\|_{2}$ and the regularization term $\mathcal{R}(\mathbf{x})$ for a fixed number of iterations $N_{\mathrm{ART}}$. In step 3 of Algorithm 1 data consistency is enforced by applying three iterations of the GPU-based Ordered Subsets-ART (OS-ART) method presented in [9]. In step 4 prior knowledge about the reconstructed volume is incorporated by applying operator $\mathcal{T}$ to the current volume estimation to reduce the penalty term $\mathcal{R}(\mathbf{x})$. All negative values in $\mathbf{x}$ are set to zero in step 5 to obtain a physically correct solution.

In this work we evaluate three different regularizers $\mathcal{R}(\mathbf{x})$. First, we investigate the iTV approach [6], where the regularization term is the total variation norm $\mathcal{R}^{\mathrm{TV}}(\mathbf{x})=$ $\|\mathbf{x}\|_{\mathrm{TV}}$. In this case operator $\mathcal{T}$ is given by an iterative gradient descent procedure with automatic adaption of the TV gradient step size to assure improved data consistency after one iteration of Algorithm 1. Second, we apply the TF Shrink method, a wavelet-based regularization approach described in [3] with $\mathcal{R}^{\mathrm{TF}}(\mathbf{x})=\|\Psi \mathbf{x}\|_{0}$, where $\Psi$ denotes a wavelet decomposition into the high pass coefficients of a redundant, piecewise linear 3D TF representation [10]. The sparsity is enforced by $\mathcal{T}$ with a soft shrinkage operation on the high pass wavelet coefficients [3]. As in [4] the high contrast vessel structures are excluded from the regularization. Third, we use the JBF regularization from [1] based on the bilateral filter (BF) [11] (ART-JBF method). The BF is a non-linear denoising filter using a combination of domain and range filtering, which is extended to the JBF by computing the range similarity using a guidance image [2]. As in [1] we use the temporal maximum intensity projection (MIP) M of the TACs as guidance image. The MIP $\mathbf{M}$ is computed as shown in steps $1-4$ of Algorithm 2: in steps 1 and 2 the baseline and contrast-enhanced sweeps are reconstructed using a short-scan FDK algorithm with a sharp Shepp-Logan filter kernel to preserve high frequent structures. In step 3 the baseline volumes are subtracted from the contrast-enhanced volumes to get pure-contrast volumes, where volumes acquired with the same rotation direction (forward or backward) are always subtracted from each other to eliminate streaking artifacts. From all pure-contrast volumes the maximum intensity of each voxel is computed. After applying a BF with range variance $\sigma_{\mathrm{R} 0}^{2}$ and domain variance $\sigma_{\mathrm{D}}^{2}$ in step 4 to reduce noise, the guidance volume $\mathbf{M}$ is obtained, which properly describes the different structures of contrast flow (such as vessels as well as healthy and stroke-affected tissue) in the scanned brain. As discussed in [12] the BF initially proposed as an intuitive tool - is related to Bayesian noise removal and can be expressed with a regularization term $\mathcal{R}(\mathbf{x})$. We define the regularization operator $\mathcal{T}$ as one application of the JBF, $\mathcal{T}(\mathbf{x})=\operatorname{JBF}\{\mathbf{x}\}_{\sigma_{\mathrm{D}}, \sigma_{\mathrm{R}}}$, with range variance $\sigma_{\mathrm{R}}^{2}$ and domain variance $\sigma_{\mathrm{D}}^{2}$.

\subsection{Iterative Denoising in Volume Space}

The ART-based algorithms exhibit a high computational complexity due to the repeated forward and back projections. Thus we introduce and evaluate the FDK-JBF method (Algorithm 2) as a computationally much faster volume-based denoising strategy: in steps 1-2 an FDK reconstruction with a sharp filter kernel of the baseline and the contrast-enhanced sweeps is applied to compute the MIP in steps 3-4 and to create initial volumes with high data consistency. Then $N_{\mathrm{V}}$ iterations (step 5) are carried out, applying the JBF on all reconstructed volumes (step 6), followed by recomputing $\mathbf{M}$ from the filtered volumes (step 7).

\section{MATERIALS \& METHODS}

To evaluate the denoising approaches a realistic digital 4D brain perfusion phantom $[13,4]$ is used. The digital brain phantom based on MR data does not have the sparse structures of classical CT phantoms, which favor algorithms apply- 


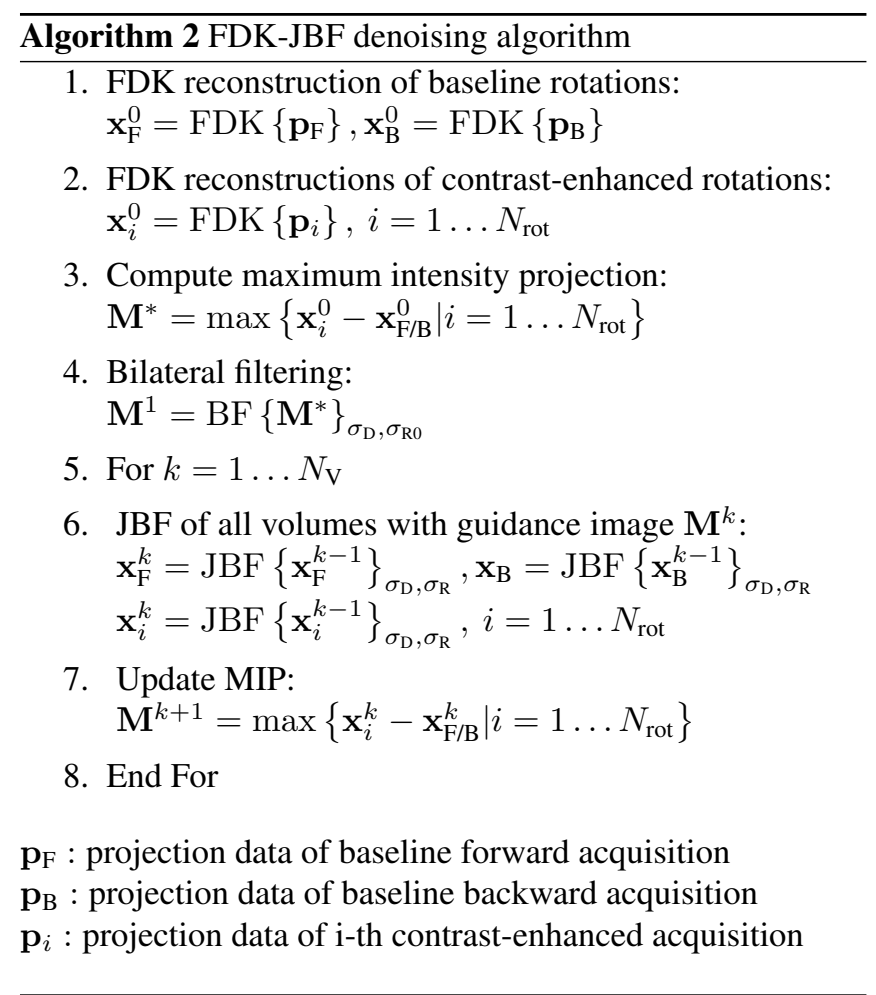

ing typical non-linear regularizers. Consequently, our brain phantom allows for a realistic numerical evaluation of the denoising algorithms. Ellipsoid ROIs simulating tissue with reduced and severely reduced perfusion were annotated in the brain phantom (see [4] for details). We created dynamic Carm projection data by forward projection according to the rapid scanning protocol. Afterwards Poisson distributed noise was added to the projection data simulating an emitted X-ray density of $3.3 \cdot 10^{5}$ photons per $\mathrm{mm}^{2}$ at the detector and a monochromatic photon energy of $60 \mathrm{keV}$ corresponding to a system dose of about $0.56 \mu \mathrm{Gy} /$ projection. The projection data was reconstructed using the different approaches discussed in Section 2 with the parameters shown in Table 2 in the appendix and $\sigma_{\mathrm{R} 0}=80 \mathrm{HU}, \sigma_{\mathrm{R}}=20 \mathrm{HU}, \sigma_{\mathrm{D}}=$ 1.5 voxels, and $N_{\mathrm{ART}}=8$. Then baseline volumes were subtracted from the contrast-enhanced volumes to create the pure-contrast volumes. Afterwards contrast TACs were sampled with a temporal resolution of $1 \mathrm{~s}$ by linear interpolation between the pure-contrast volumes. The CBF, CBV, and MTT maps were calculated from the TACs using the truncated SVD algorithm [14]. For quantitative evaluation the root mean square error (RMSE) over time between the reconstructed and the ground-truth TACs was computed for the arterial input function (AIF) and the TACs of the brain tissue. To evaluate the reconstructed perfusion maps, reference perfusion maps were created from the ground truth TACs. We calculated the Pearson correlation (PC) between the reconstructed and the reference maps by applying an automated ROI analysis: the maps were partitioned slice-wise into quadratic areas of $4 \times 4$

\begin{tabular}{c|c|c|c|c|c} 
& FDK & iTV & TFShrink & ART-JBF & FDK-JBF \\
\hline PC CBF & 0.61 & 0.80 & 0.79 & $\mathbf{0 . 8 1}$ & 0.79 \\
PC CBV & 0.55 & 0.75 & 0.76 & $\mathbf{0 . 7 7}$ & 0.76 \\
PC MTT & 0.49 & 0.74 & 0.72 & 0.78 & $\mathbf{0 . 8 1}$ \\
PC TTP & 0.55 & 0.74 & 0.73 & $\mathbf{0 . 7 8}$ & 0.77 \\
\hline CT & $\mathbf{1}$ & 36 & 39 & 30 & 3
\end{tabular}

Table 1. Quantitative results of digital brain phantom study (CT: computation time).

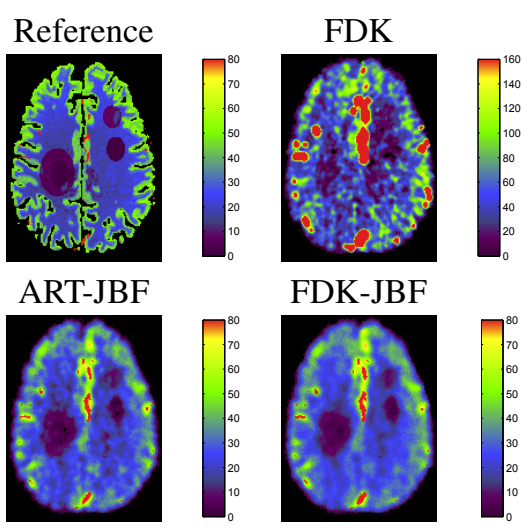

Fig. 1. Reconstructed CBF perfusion maps from digital brain perfusion phantom data (units: $\mathrm{ml} / 100 \mathrm{~g} / \mathrm{min}$ ).

pixels and the average perfusion values of the ROIs belonging to the brain tissue were used as measurement variables for the PC computation. ROIs containing vascular structures or air were ignored. The FDK and FDK-JBF techniques were also used to reconstruct 3D perfusion maps from an animal study, where an ischemic stroke was induced in a healthy canine under an institutionally approved protocol and a PCCT acquisition was conducted using the rapid scanning protocol.

\section{RESULTS}

Table 1 shows the quantitative comparison between the reconstruction algorithms with respect to PC and the total computation time for reconstruction of all volumes. For more detailed results also including the RMSE of the reconstructed TACs see Table 2 in the appendix. The volumes had a size of $256 \times 256 \times 86$ voxels and were reconstructed on a laptop computer with an Intel i7 M 6202 x $2.7 \mathrm{GHz}$ CPU, 8 GB RAM, and an Nvidia Quadro FX 880M graphics chip set. Figure 1 illustrates the reconstructed CBF perfusion maps using FDK, ART-JBF, and FDK-JBF algorithms. The CBV, MTT, and TTP maps are shown Figure 3 in the appendix. The 3D canine study CBF perfusion maps are shown in Figure 2. Additionally the TTP maps are shown in Figure 4 of the appendix. 


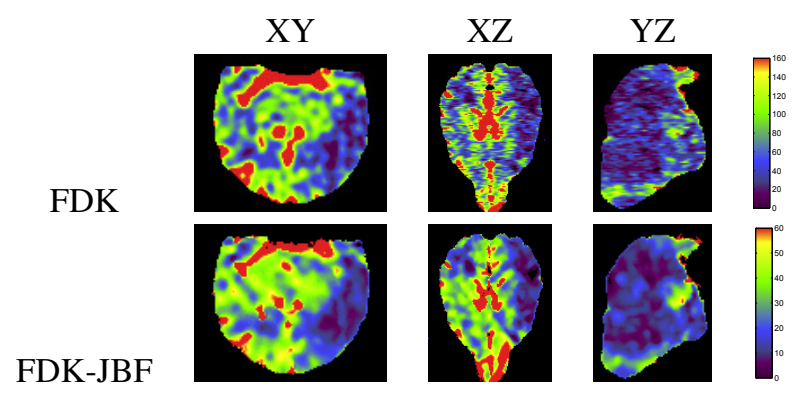

Fig. 2. 3D CBF perfusion maps from canine stroke model (units: $\mathrm{ml} / 100 \mathrm{~g} / \mathrm{min}$ ).

\section{DISCUSSION \& CONCLUSIONS}

The quantitative results in Table 1 and Table 2 show that all denoising strategies improve the RMSE of reconstructed TACs and the PC of the reconstructed perfusion maps compared to native FDK reconstruction. The best results are achieved using the JBF-based approaches, where the FDKJBF result is very close to the ART-JBF result with a computation time reduced by a factor of ten. Comparing the perfusion maps shown in Figure 1 and Figure 3 reconstructed with the JBF approaches to the FDK approach, the JBF approaches show several advantages: the stroke-affected areas are much better separated from the healthy tissue and their shape is much closer to their shape in the reference maps. Also the area of increased perfusion values around the vessels in the CBF and CBV maps (red pixels) due to smoothing is much higher in the FDK than in the JBF maps. The perfusion values in the brain tissue are in a range of $20-60 \mathrm{ml} / 100 \mathrm{~g} / \mathrm{min}$ for CBF resp. 2-6 ml/100g for CBV in the JBF maps which is the same range as in the reference maps, but the range in the FDK maps is $40-120 \mathrm{ml} / 100 \mathrm{~g} / \mathrm{min}$ for CBF resp. $4-12 \mathrm{ml} / 100 \mathrm{~g}$ for CBV. This also holds for the 3D canine CBF maps from real data shown in Figure 2: the vessels are less blurred and the perfusion value range is closer to physiologically expected values in the FDK-JBF CBF map than in the FDK CBF map. Furthermore the FDK-JBF maps are smoother in $\mathrm{XZ}$ and $\mathrm{YZ}$ viewing directions.

In this work we evaluated the use of different denoising methods for perfusion $\mathrm{C}$-arm $\mathrm{CT}$ with a rapid scanning protocol. All evaluated methods showed a perceptible improvement compared to standard FDK-type reconstruction. However, using ART-based approaches this comes for the price of a much higher computational effort. Thus we introduced a pure volume-based denoising technique with much smaller computational complexity and showed that it performs similarly to the ART-based approaches. One problem of JBFbased approaches might be streak artifacts, which cannot be handled well by a bilateral filter. Due to the low number of 133 projections they appear in the reconstructed volumes but vanish after subtraction of the baseline volumes. However, in real patient acquisitions they might not be always repro- ducible and the elimination of such artifacts represents a direction of future research. Furthermore hardware improvements involving rotation speed and detector read out rate are also worth of investigation.

\section{Acknowledgments}

We would like to thank Dr. Charles Strother and his research team at the University of Wisconsin-Madison for contributing the experimental canine ischemic perfusion study data.

\section{REFERENCES}

[1] M. Manhart, M. Kowarschik, A. Fieselmann, Y. Deuerling-Zheng, and J. Hornegger, "Fast dynamic reconstruction algorithm with joint bilateral filtering for perfusion C-arm CT," in IEEE NSS MIC Conference Record, Anaheim, USA, 2012, pp. 2304-2311.

[2] G. Petschnigg, M. Agrawala, H. Hoppe, R. Szeliski, M. Cohen, and K. Toyama, "Digital photography with flash and no-flash image pairs," in Proc. ACM SIGGRAPH, 2004, pp. 664-672.

[3] X. Jia, B. Dong, Y. Lou, and S. B. Jiang, "GPU-based iterative conebeam CT reconstruction using tight frame regularization," Physics in Medicine and Biology, vol. 56, no. 13, pp. 3787-3807, 2011.

[4] M. Manhart, A. Fieselmann, and Y. Deuerling-Zheng, "Evaluation of a tight frame reconstruction algorithm for perfusion C-arm CT using a realistic dynamic brain phantom," in Proc. of the Second International Conference on Image Formation in X-Ray Computed Tomography, Salt Lake City, USA, 2012, pp. 123-126.

[5] L. Feldkamp, L. Davis, and J. Kress, "Practical cone-beam algorithm," Journal of the Optical Society of America A, vol. 1, no. 6, pp. 612-619, 1984.

[6] L. Ritschl, F. Bergner, C. Fleischmann, and M. Kachelrieß, "Improved total variation-based CT image reconstruction applied to clinical data," Physics in Medicine and Biology, vol. 56, no. 6, pp. 1545-1561, 2011.

[7] A. Kak and M. Slaney, Principles of computerized tomographic imaging. Piscataway, NJ: IEEE Service Center, 1988.

[8] H. Bruder, R. Raupach, J. Sunnegardh, M. Sedlmair, K. Stierstorfer, and T. Flohr, "Adaptive iterative reconstruction," Proc. SPIE Med. Img.: Physics of Medical Imaging, vol. 7961, pp. 79610J-1, 2011.

[9] B. Keck, H. Hofmann, H. Scherl, M. Kowarschik, and J. Hornegger, "GPU-accelerated SART reconstruction using the CUDA programming environment," in Proc. SPIE Med. Img.: Physics of Medical Imaging, vol. 7258, 2009, pp. 72 582B-1.

[10] B. Dong and Z. Shen, "MRA-based wavelet frames and applications," IAS Lecture Notes Series, Summer Program on "The Mathematics of Image Processing", Tech. Rep., 2010.

[11] C. Tomasi and R. Manduchi, "Bilateral filtering for gray and color images," in Proc. 6th IEEE ICCV, 1998, pp. 839-846.

[12] M. Elad, "On the origin of the bilateral filter and ways to improve it," IEEE Trans Img Proc, vol. 11, pp. 1141-1151, 2002.

[13] A. J. Riordan, M. Prokop, M. A. Viergever, J. W. Dankbaar, E. J. Smit, and H. W. A. M. de Jong, "Validation of CT brain perfusion methods using a realistic dynamic head phantom," Medical Physics, vol. 38, no. 6, pp. 3212-3221, 2011.

[14] A. Fieselmann, M. Kowarschik, A. Ganguly, J. Hornegger, and R. Fahrig, "Deconvolution-based CT and MR brain perfusion measurement: Theoretical model revisited and practical implementation details," International Journal of Biomedical Imaging, 2011, article ID 467563. 


\begin{tabular}{c|ccc|c|c|c|ccc} 
& \multicolumn{3}{|c|}{ FDK } & iTV & TF Shrink & ART-JBF & \multicolumn{3}{c}{ FDK-JBF } \\
\hline Parameters & $\sigma_{\mathrm{K}}=1.0$ & $\sigma_{\mathrm{K}}=1.25$ & $\sigma_{\mathrm{K}}=1.5$ & $N_{\mathrm{TV}}=3$ & $\mu=20$ & & $N_{\mathrm{V}}=1$ & $N_{\mathrm{V}}=3$ & $N_{\mathrm{V}}=4$ \\
\hline \hline RMSE AIF & 121.40 & 143.85 & 165.70 & 35.83 & 35.72 & $\mathbf{2 7 . 7 3}$ & 47.34 & 47.34 & 47.34 \\
RMSE Tissue & 3.87 & 3.24 & 2.97 & 2.55 & 2.53 & 1.99 & 2.25 & $\mathbf{1 . 9 8}$ & 2.03 \\
\hline PC CBF & 0.60 & 0.61 & 0.60 & 0.80 & 0.79 & $\mathbf{0 . 8 1}$ & 0.78 & 0.79 & 0.76 \\
PC CBV & 0.53 & 0.55 & 0.55 & 0.75 & 0.76 & $\mathbf{0 . 7 7}$ & 0.74 & 0.76 & 0.73 \\
PC MTT & 0.43 & 0.49 & 0.53 & 0.74 & 0.72 & 0.78 & 0.72 & $\mathbf{0 . 8 1}$ & $\mathbf{0 . 8 1}$ \\
PC TTP & 0.47 & 0.55 & 0.61 & 0.74 & 0.73 & $\mathbf{0 . 7 8}$ & $\mathbf{0 . 7 8}$ & 0.77 & 0.76 \\
\hline CT & $\mathbf{1}$ & $\mathbf{1}$ & $\mathbf{1}$ & 36 & 39 & 30 & 2 & 3 & 3
\end{tabular}

Table 2. Quantitative results of digital brain phantom study $\left(\sigma_{\mathrm{K}}\right.$ : smoothness of FDK filter kernel, $N_{\mathrm{TV}}$ : TV minimization iterations in each regularization step, $\mu$ : soft-shrinkage threshold, $N_{\mathrm{V}}$ : number of denoising iterations in volume space, RMSE: root mean square error over time in HU, PC: Pearson correlation, CT: computation time in minutes, bold numbers indicate best result in row).

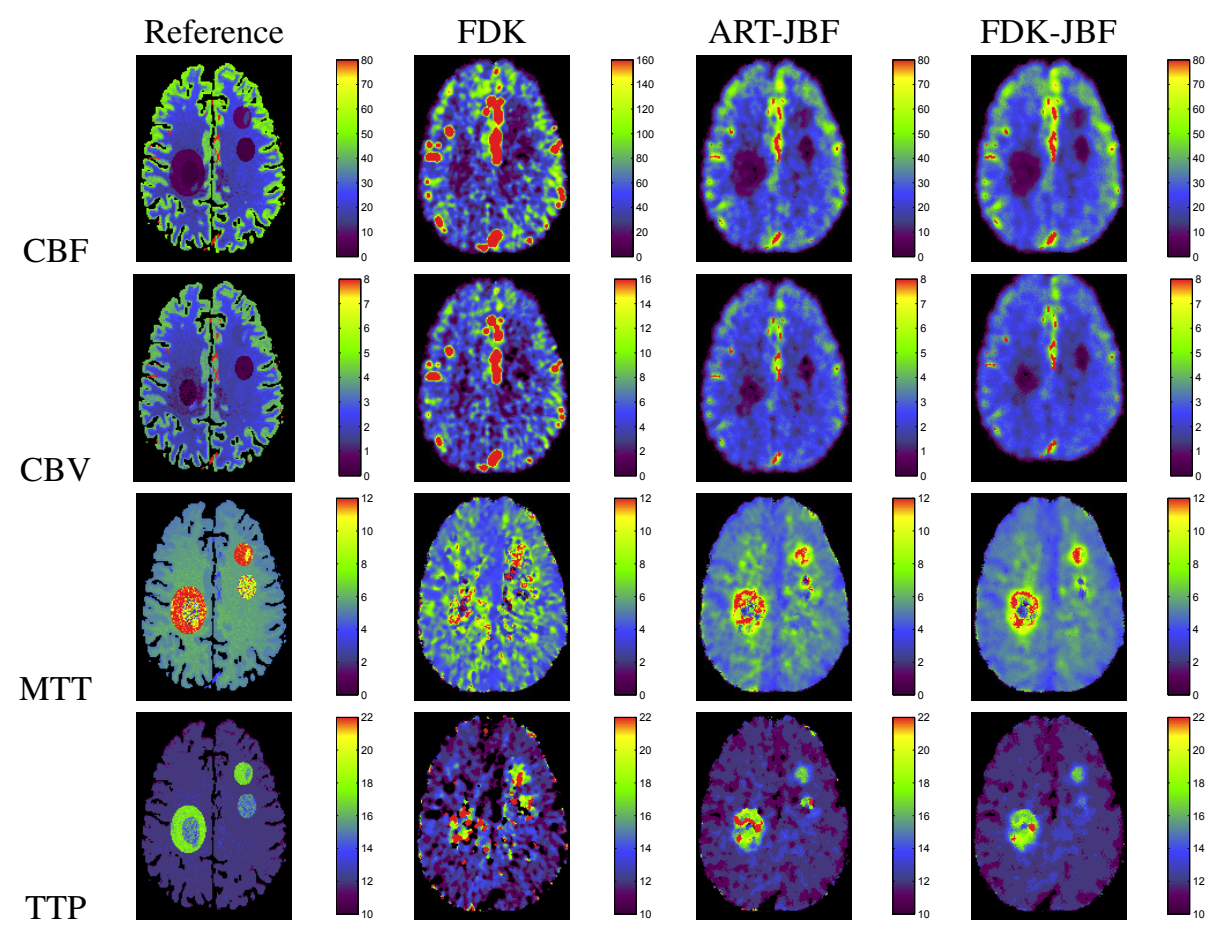

Fig. 3. CBF (units: $\mathrm{ml} / 100 \mathrm{~g} / \mathrm{min}$ ), CBV (units: $\mathrm{ml} / 100 \mathrm{~g}$ ), MTT (units: $\mathrm{s}$ ), and TTP (units: s) perfusion maps from digital brain perfusion phantom data reconstructed with FDK $\left(\sigma_{\mathrm{K}}=1.25\right)$, ART-JBF, and FDK-JBF $\left(N_{\mathrm{V}}=3\right)$ algorithms.

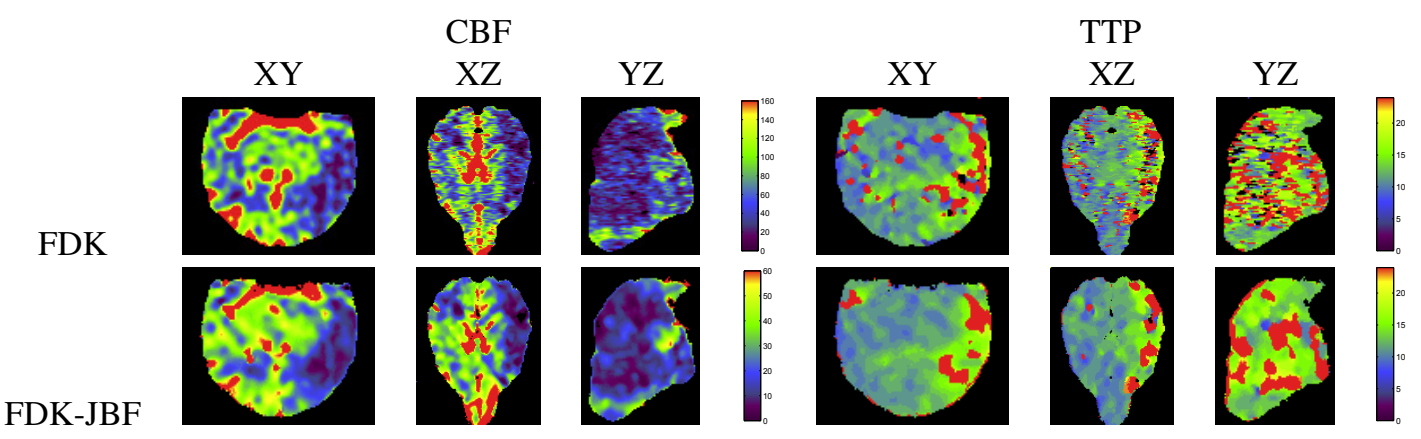

Fig. 4. 3D CBF (units: $\mathrm{ml} / 100 \mathrm{~g} / \mathrm{min}$ ) and TTP (units: s) perfusion maps from canine stroke model. 